\title{
Determination of force-free wet adhesion of mussel-inspired polymers to spin labeled surface
}

\author{
İklima Kırpat ${ }^{a}$, Yaman Göksel ${ }^{a}$, Erman Karakuş ${ }^{b}$, Mustafa Emrullahoğlu ${ }^{b}$, Yasar Akdogan ${ }^{a, *}$ \\ ${ }^{a}$ Materials Science and Engineering Department, Izmir Institute of Technology, 35430 Urla, Izmir, Turkey \\ ${ }^{\mathrm{b}}$ Department of Chemistry, Izmir Institute of Technology, 35430 Urla, İmir, Turkey
}

\section{A R T I C L E I N F O}

\section{Article history:}

Received 16 May 2017

Received in revised form 9 June 2017

Accepted 11 June 2017

Available online 13 June 2017

\section{Keywords:}

Adhesion

Biomimetic

EPR spectroscopy

Hydration layers

DOPA

\begin{abstract}
A B S T R A C T
Hydration repulsive forces oppose the adhesive interactions, especially in the force-free conditions. Here, we studied spontaneous wet adhesion of 3,4-dihydroxyphenylalanine (DOPA) functionalized poly(ethylene glycol) (PEG) polymers inspired by marine mussels. Using electron paramagnetic resonance (EPR) spectroscopy, we can monitor spontaneous adhesion of DOPA containing polymer to suspended spin labeled hydrophobic polystyrene nanobeads at molecular level. The surface coverage up to $82 \%$ is obtained from EPR measurements. However, in the force-free condition, EPR measurements do not show any detectable DOPA based adhesion to hydrophilic silica nanobead.
\end{abstract}

(c) 2017 Elsevier B.V. All rights reserved.

\section{Introduction}

Marine mussels have exceptional underwater adhesive abilities. Their adhesive mechanisms have become the focus of much attention for designing artificial wet adhesives. Mussels attach to various types of surfaces by seven known mussel foot proteins (Mfps). All Mfps are containing posttranslationally modified DOPA which plays a critical role in the adhesion of Mfps to wet surfaces $[1,2]$.

In order to achieve the wet adhesion, mussels apply force to disturb the hydration layers and/or mussels dry the surface using Mfps [3]. Similarly, most of the studies e.g. surface force apparatus (SFA) and atomic force microscope (AFM) measurements were applied with external force to achieve durable adhesion in water $[4,5]$. However, various spectroscopic techniques can measure how well Mfps adhere to surfaces under force-free conditions [6-9]. All of these techniques have provided invaluable information about the molecular mechanism of mussel adhesion, which is achieved mainly by DOPA.

DOPA molecules have been incorporated into several polymers, including PEG, to obtain wet adhesive synthetic materials [10-13]. Here, we employed the EPR spectroscopy to assess the adhesion of a DOPA modified 4-armed PEG polymer (Fig. 1(A)) in the force-free condition. As model surfaces we prepared spin labeled hydropho-

\footnotetext{
* Corresponding author.

E-mail address: yasarakdogan@iyte.edu.tr (Y. Akdogan).
}

bic polystyrene (SL-PS) and hydrophilic silica $\left(\mathrm{SL}_{-} \mathrm{SiO}_{2}\right)$ nanobeads suspended in solution (Fig. 1(B)). Furthermore, we can calculate fraction of the nanobead surface covered by adhesives using EPR.

\section{Experimental}

The synthesis of PEG-(N-Boc-L-DOPA $)_{4}$ and PEG-(Trp) $)_{4}$ were prepared as described in published procedures and explained in supplementary file [14-16]. For PEG-(N-Boc-L-DOPA), briefly, the amine group of L-DOPA was protected with tertbutyloxycarbonyl (Boc) group and then the product of N-Boc-L-DOPA was reacted with PEG-( $\left.\mathrm{NH}_{2}\right)_{4}(10 \mathrm{kDa})$. The DOPA-modified PEG molecules were purified by precipitation in cold diethyl ether and examined using ${ }^{1} \mathrm{H}$ NMR spectroscopy, UV-vis spectroscopy and ninhydrin test (Figs. S1-S3). For PEG-(Trp) $)_{4}$, briefly, PEG-(OH) $4(10 \mathrm{kDa})$ and p-nitrophenyl chloroformate were mixed to obtain PEG-(p-nitrophenyl carbonate) 4 . Next, tryptophan and PEG-(p-nitrophenyl carbonate $)_{4}$ were mixed to get PEG-(Trp) $)_{4}[16] .{ }^{1} \mathrm{H}$ NMR data are available in SI (Fig. S4).

SL-PS and $\mathrm{SL}_{-} \mathrm{SiO}_{2}$ nanobeads were prepared according to previous study [9]. Amine modified polystyrene and silica nanobeads ( $\sim 60 \mathrm{~nm})$ were mixed with 4-carboxy-Tempo and 1-ethyl-3-(3dimethylaminopropyl) carbodiimide in MES buffer ( $\mathrm{pH}$ 3.0) for one day at room temperature (RT). The resulting SL-PS and $\mathrm{SL}-\mathrm{SiO}_{2}$ were purified in centrifuge concentrator with washing several times with MES buffer. SL-PS and $\mathrm{SL}_{-} \mathrm{SiO}_{2}$ were mixed with different concentrations of PEG-( $\left.\mathrm{NH}_{2}\right)_{4}$ and PEG-(N-Boc-L-DOPA $)_{4}$ 
(A)
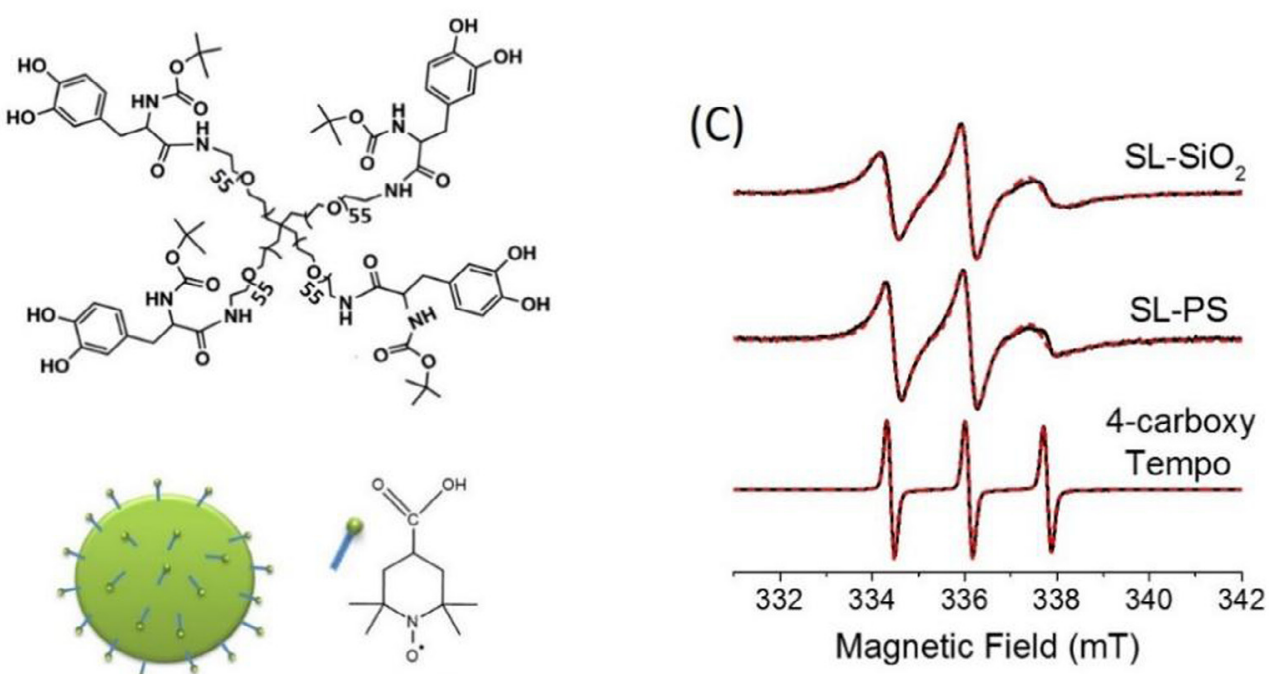

(B)
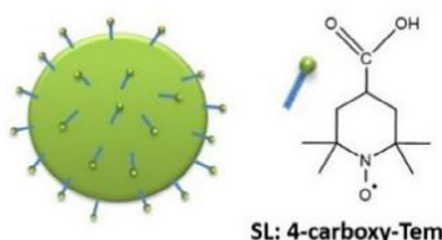

SL: 4-carboxy-Tempo

SL-PS or SL-SiO

Fig. 1. (A) Chemical structure of the used PEG-(N-Boc-L-DOPA) $)_{4}$. (B) SL-PS or SL-SiO ${ }_{2}$ nanobeads. Pins represent SL, 4-carboxy-Tempo. (C) EPR spectra of 4-carboxy-Tempo, SL$\mathrm{PS}$ and $\mathrm{SL}_{-} \mathrm{SiO}_{2}$ (black) and their simulations (red). (For interpretation of the references to colour in this figure legend, the reader is referred to the web version of this article.)
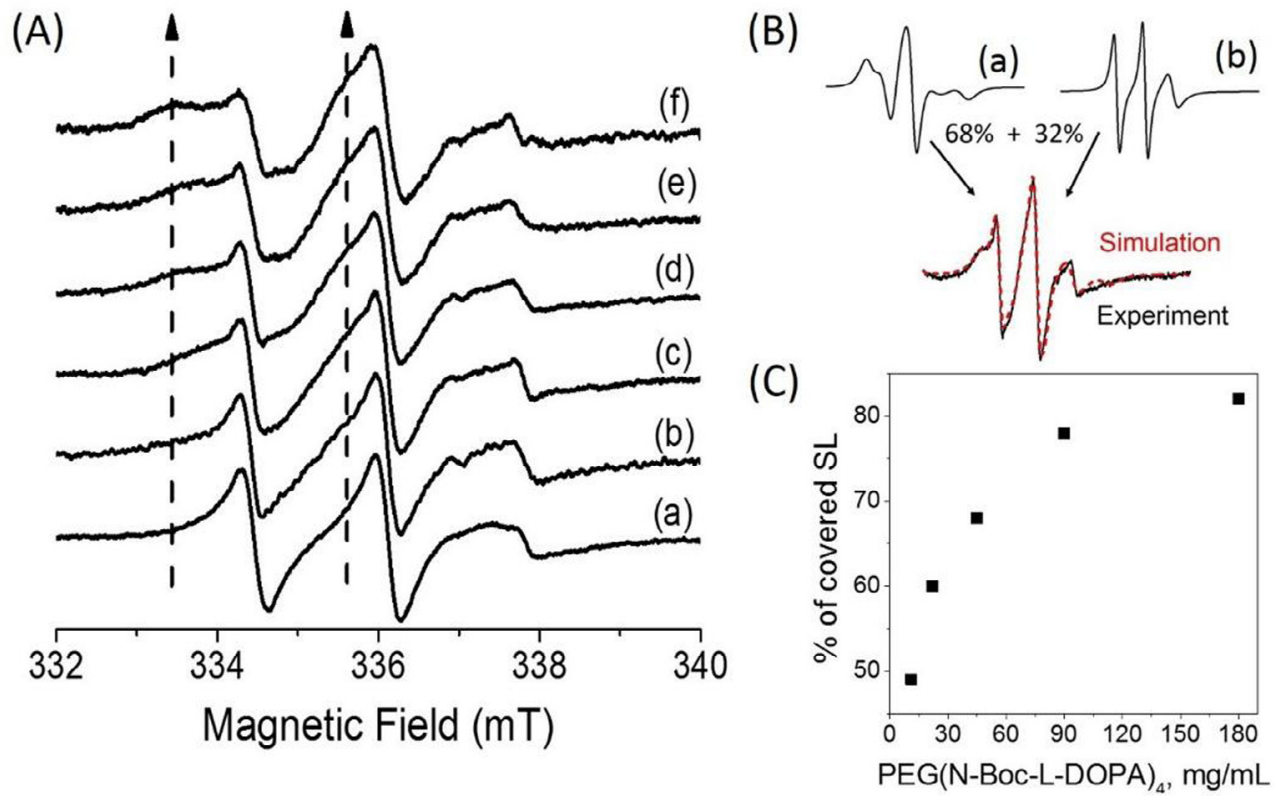

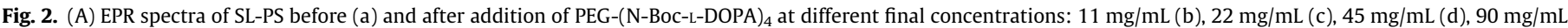

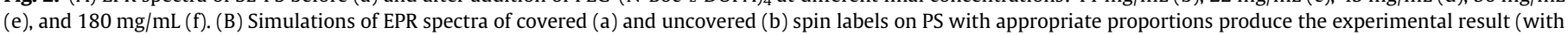
$45 \mathrm{mg} / \mathrm{mL}$ PEG-(N-Boc-L-DOPA $)_{4}$ ). (C) Determination of the percentage of covered SL on PS upon addition of the PEG-(N-Boc-L-DOPA) . $^{2}$

with a volume ratio of 1:1 in MES buffer at $\mathrm{pH}$ 3.0. Acidic medium was used to avoid DOPA oxidation.

X-band EPR measurements were done using a CMS 8400 (Adani) benchtop spectrometer at RT. All spectra were normalized to the intensities of the middle signals and also they were simulated using the Matlab-based Easyspin 4.5.5 software package [17].

\section{Results and discussion}

Fig. 1(C) shows EPR spectra of SL-PS and $\mathrm{SL}_{-} \mathrm{SiO}_{2}$ in solution with their corresponding simulations. For comparison, reference spectrum of 4-carboxy-Tempo (spin label) is displayed. In solution, rotational dynamics of spin labels can be studied by the analysis of
EPR line shapes [18,19]. At RT, the 4-carboxy-Tempo possesses sharp three-line signals with a rotational correlation time $\tau_{R}=2.0 \mathrm{ps}$, signatures of freely tumbling motion. However, EPR line shapes of SL-PS and $\mathrm{SL}_{-} \mathrm{SiO}_{2}$ consist of broad hyperfine lines stemming from restricted rotational motion, with $\tau_{R} 2.8 \mathrm{~ns}$ and $2.9 \mathrm{~ns}$, respectively. These results show that binding of spin labels to the surface of nanobeads restricts the rotational freedom and increases the rotational correlation time range from the ps to the ns range.

Fig. 2(A) shows EPR spectra of SL-PS before and after addition of PEG-(N-Boc-DOPA) 4 with different concentrations from $11 \mathrm{mg} / \mathrm{mL}$ to $180 \mathrm{mg} / \mathrm{mL}$. Explicitly, a second type of signal with a longer rotational correlation time is observed upon addition of 


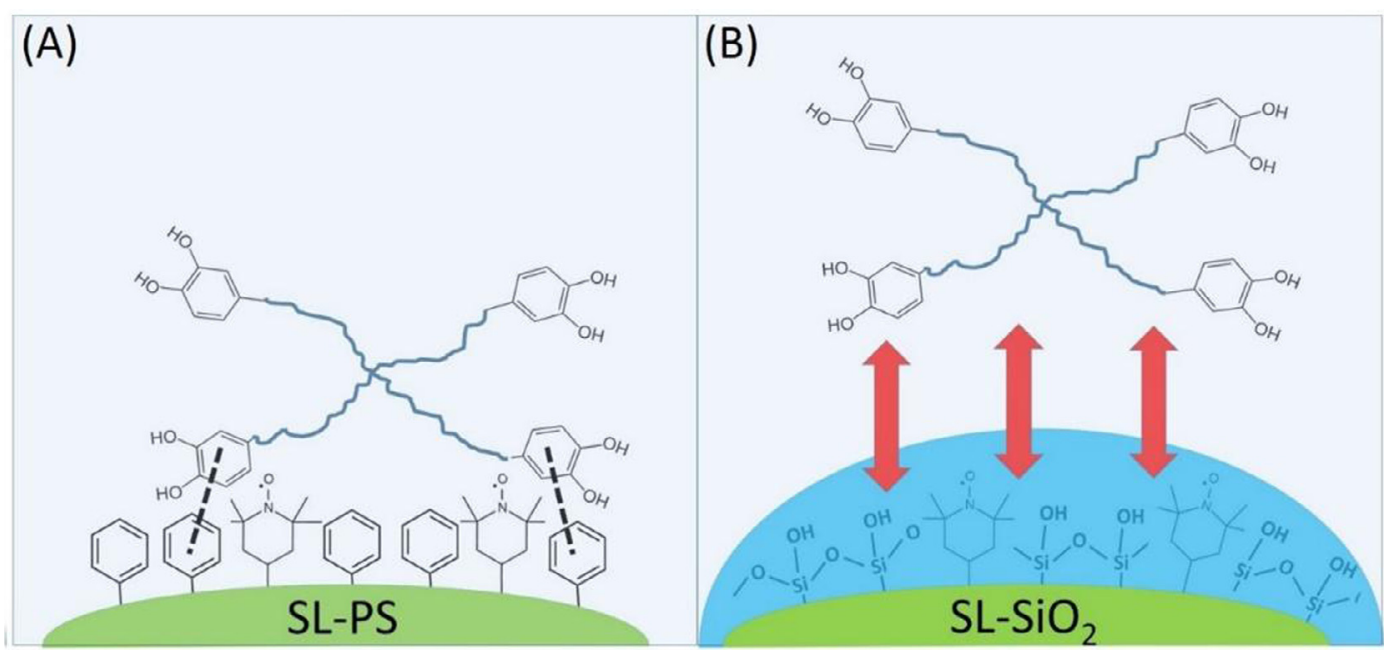

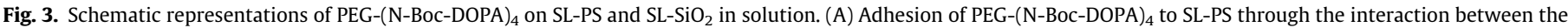

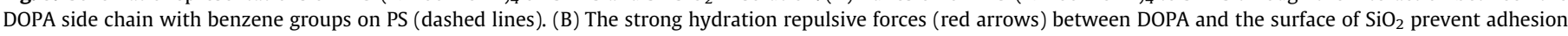

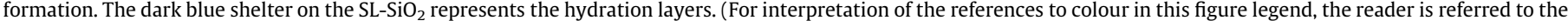
web version of this article.)

PEG-(N-Boc-DOPA) 4 . The simulated spectra reveal two types of signals with different $\tau_{R}$ values: the shorter $\tau_{R}$ value with $2.8 \mathrm{~ns}$ belongs to spin labels on the uncovered PS, and the longer $\tau_{R}$ value with 10 ns belongs to spin labels which are affected by PEG-(NBoc-DOPA $)_{4}$ adhesion (Fig. S5 and Fig. 2(B)). Obtaining longer rotational correlation time indicates that some of the spin labels on the PS nanobeads are covered with PEG-(N-Boc-DOPA) 4 .

Furthermore, as explained in more detail in SI, simulation of the EPR spectrum of SL-PS after addition of PEG-(N-Boc-DOPA) 4 provides the percentage of covered and uncovered spin labels on PS. The sum of EPR spectra of simulated individual spin labels with an appropriate ratio yields the experimental result. Accordingly, in the presence of $45 \mathrm{mg} / \mathrm{mL}$ PEG-(N-Boc-DOPA) 4 , the percentages of covered and uncovered spin labels on PS were found $68 \%$ and $32 \%$, respectively (Fig. 2(B)). This shows that PEG-(N-Boc-DOPA) 4 adhere to PS surface significantly. After simulations of all EPR spectra of SL-PS and PEG-(N-Boc-DOPA $)_{4}$ conjugations, we observed that $49 \%$ and $82 \%$ of the spin labels on the PS are covered upon addition of $11 \mathrm{mg} / \mathrm{mL}$ and $180 \mathrm{mg} / \mathrm{mL}$ PEG-(N-Boc-DOPA) 4 , respectively (Fig. 2(C) and Fig. S5).

We tested the same experiment with $90 \mathrm{mg} / \mathrm{mL}$ PEG- $\left(\mathrm{NH}_{2}\right)_{4}$ and PEG-(Trp $)_{4}$ (Fig. S6). EPR spectra of SL-PS before and after addition of PEG-( $\left.\mathrm{NH}_{2}\right)_{4}$ or PEG-(Trp $)_{4}$ are almost same which indicates that they do not significantly adhere to the surface of SL-PS in solution. This emphasizes the necessity of DOPA in this polymer-PS conjugation. In the literature, adhesion of DOPA to both organic and inorganic surfaces have been explained by hydrophobic interaction, hydrogen bonding, electrostatic interaction, cation- $\pi$ interaction, and $\pi-\pi$ interaction [20]. Since PS nanobead has hydrophobic benzene rings on the surface, DOPA adhere to the PS surface via hydrophobic and $\pi-\pi$ interactions (Fig. 3) [9,20]. By protecting the amino group of DOPA with the Boc group, the potential electrostatic interaction between DOPA and PS are eliminated.

To gain more detailed insight into the adhesion of DOPA to different surfaces, we studied the adhesion of PEG-(N-Boc-DOPA $)_{4}$ to hydrophilic surface of $\mathrm{SL}_{-} \mathrm{SiO}_{2}$. Fig. S7 shows EPR spectra of $\mathrm{SL}_{-} \mathrm{SiO}_{2}$

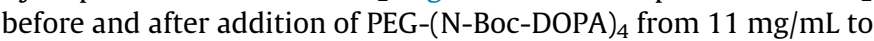
$180 \mathrm{mg} / \mathrm{mL}$. The EPR spectrum of $\mathrm{SL}_{-} \mathrm{SiO}_{2}$ is not affected notably upon addition of PEG-(N-Boc-DOPA $)_{4}$. In the literature, SFA measurements showed that Mfp-1, Mfp-3 and Mfp-5 adhere much stronger to hydrophobic surfaces than to hydrophilic surfaces [4]. This shows the significance of hydrophobic interactions for the strong adhesion while the hydrophilic interactions are still adequate for the relatively weak adhesion [4]. Lu et al. showed that stronger applied force and longer waiting time promote the adhesion between DOPA and $\mathrm{SiO}_{2}$ [20]. However, we did not observe DOPA based adhesion to hydrophilic $\mathrm{SiO}_{2}$ surface. This can be explained by the existence of strong hydration layers around the $\mathrm{SiO}_{2}$. Without applying force, the comparably weak hydrophilic forces cannot overcome the hydration repulsive forces around the $\mathrm{SiO}_{2}$ (Fig. 3) [9].

\section{Conclusion}

In this study, unlike the DOPA adhesion studies in the literature, we look at spontaneous DOPA adhesion in solution without applying any force. EPR spectroscopy allows us to detect the adhesion at molecular level because spin labels on the surface are directly affected by the macromolecule binding. We showed that hydration layers are important for the force-free wet adhesion. Since the hydration layers form double barriers between DOPA and hydrophilic $\mathrm{SiO}_{2}$ surface, adhesion formation is hindered. In contrast, hydrophobic and $\pi-\pi$ interactions are strong enough to overcome the weak repulsive hydration layers between DOPA and PS surface. So, PEG-(N-Boc-DOPA $)_{4}$ is able to cover up to $82 \%$ of PS surface.

\section{Acknowledgements}

This work was financially supported by Turkish Scientific and Technological Research Council (Tubitak) via 3501 Program under grant $114 Z 318$.

\section{Appendix A. Supplementary data}

Supplementary data associated with this article can be found, in the online version, at http://dx.doi.org/10.1016/j.matlet.2017.06. 060 .

\section{References}

[1] B.P. Lee, P.B. Messersmith, J.N. Israelachvili, H. Waite, Annu. Rev. Mater. Res. 41 (2011) 99-132.

[2] P.K. Forooshani, B.P. Lee, J. Polym. Sci. A 55 (2017) 9-33.

[3] N.R.M. Rodriguez, S. Das, Y. Kaufman, J.N. Israelachvili, J.H. Waite, Biofouling 31 (2015) 221-227. 
[4] J. Yu, Y. Kan, M. Rapp, E. Danner, W. Wei, S. Das, D.R. Miller, Y. Chen, J.H. Waite, J.N. Israelachvili, Proc. Natl. Acad. Sci. U.S.A. 110 (2013) 15680-15685.

[5] H. Lee, N.F. Scherer, P.B. Messersmith, Proc. Natl. Acad. Sci. U.S.A. 103 (2006) 12999-13003.

[6] Y. Kan, E.W. Danner, J.N. Israelachvili, Y. Chen, J.H. Waite, PLoS One 9 (2014) e108869.

[7] A.A. Ooka, R.L. Garrell, Biopolymers (Biospectroscopy) 57 (2000) 92-102.

[8] W. Wei, L. Petrone, Y. Tan, H. Cai, J.N. Israelachvili, A. Miserez, J.H. Waite, Adv. Funct. Mater. 26 (2016) 3496-3507.

[9] Y. Akdogan, W. Wei, K.Y. Huang, Y. Kageyama, E.W. Danner, D.R. Miller, N.R.M Rodriguez, J.H. Waite, S. Han, Angew. Chem. Int. Ed. 53 (2014) 11253-11256.

[10] Y. Liu, H. Meng, S. Konst, R. Sarmiento, R. Rajachar, B.P. Lee, ACS Appl Mater Interfaces 6 (2014) 16982-16992.

[11] Y. Li, H. Meng, Y. Liu, A. Narkar, B.P. Lee, ACS Appl Mater. Interfaces 8 (2016) $11980-11989$.

[12] G. Westwood, T.N. Horton, J.J. Wilker, Macromolecules 40 (2007) 3960-3964.
[13] L. Li, Y. Li, X.F. Luo, J.P. Deng, W.T. Yang, React. Funct. Polym. 70 (2010) 938943.

[14] G. Giorgioni, F. Claudi, S. Ruggieri, M. Ricciutelli, G.F. Palmieri, A. Di Stefano, P. Sozio, L.S. Cerasa, A. Chiavaroli, C. Ferrante, G. Orlando, Bioorg Med. Chem. 18 (2010) 1834-1843.

[15] B.P. Lee, J.L. Dalsin, P.B. Messersmith, Biomacromolecules 3 (2002) 1038-1047.

[16] C. Mueller, M.A.H. Capelle, T. Arvinte, E. Seyrek, G. Borchard, Eur. J. Pharm. Biopharm. 79 (2011) 646-657.

[17] S. Stoll, A. Schweiger, J. Magn. Reson. 178 (2006) 42-55.

[18] D. Tatlidil, M. Ucuncu, Y. Akdogan, Phys. Chem. Chem. Phys. 17 (2015) 2267822685.

[19] Y. Akdogan, M. Emrullahoglu, D. Tatlidil, M. Ucuncu, G. Cakan-Akdogan, Phys. Chem. Chem. Phys. 18 (2016) 22531-22539.

[20] Q. Lu, E. Danner, J.H. Waite, J.N. Israelachvili, H. Zeng, D.S. Hwang, J. R. Soc. Interface 10 (2013) 20120759. 